\title{
MAGNETOSTIMULATED INHOMOGENEITY OF ELECTRIC FIELD IN ALUMINUM
}

\author{
V.R.Sobol, O.N.Mazurenko, and A.A.Drozd \\ Institute of Solid State and Semiconductor Physics ASB \\ Minsk, 220072, Belarus
}

\begin{abstract}
The peculiarities of potential and current distribution in metals under inhomogeneous action of magnetic field is studied experimentally and analytically. Magnetic fleld inhomogeneity is modeled with method of curving the electric current lines in rectangular conductors through the use of preset profiles of samples. Observed inhomogeneous distribution of electric potential is analyzed on the base of charge continuity. It is shown that current density redistribution takes place. Near one side current density is high and near unother it is small. This is a reason of decrease of an effective cross-section of conductor with respective enhancement of magnetoresistance. Some analytical relations and modes of applications of observed phenomena in cryogenic electronic devices are proposed.
\end{abstract}

\section{INTRODUCTION}

The problem of inhomogencity of electric field and current in single and composite conductors under magnetic field is discussed at present. There are many works devoted to study of inhomozeneity stimulated by charge concentration, geometry, dielectric inclusions, interface between different metals and others 10 . In spite of different nature of inhomogeneities there is the general in their influence on transport processes namely an appearance of Hall current which leads to different macroscopic displaces particularly to increase of magnetoresistance. This is a consequence of potentiality of steady electric field in inhomogeneous media when a rotation free type of field requires an appearance of curl current.

This research is dedicated to investigation of charge transport in aluminum under inhomogeneous action of external magnetic field. Some results of experimental and theoretical study of electric potential distribution and peculiarities of charge flow in rectangular samples are represented. We regard here the macroscopic samples having comparatively small electron relaxation length when local kinetic coefficient distribution is given. Local connection between electric field $E$, its potential $\varphi$ and 
current density $f$ through the sample when current $I$ is determined by power supply are analyzed.

\section{EXPERIMENTAL PROCEDURE}

To model the inhomogeneous action of magnetic fleld a method of curving the electric current lines in rectangular aluminum conductor is used". For this the respective plate's profiles are chosen for local normal projections $B_{1}$ of an external magnetic field $B$ to vary regularly along transport direction in accordance with preset linear or exponential law (Fig. la,b). An external magnetic field coincides with $z$-axis. Sample profile in $\mathrm{ZX}$ plane corresponds to $x^{\prime}$ line. Current is made to flow along $x^{\prime}$. Local effective magnetic field is $B_{1}=B_{0}+K^{\prime} x^{\prime}$ or $B_{1}=B_{0} \exp \left(k x^{\prime}\right)$. Here $B_{0}$ is a local normal projection of magnetic field at $x^{\prime}=0, K^{\top}$ and $k$ are magnetic field gradients for linear and exponential types of inhomogeneity. In Fig. Ic the sample having a width $b$ is presented in plane $Y X$, hereinafter $x^{\prime}$ is denoted as $x$. For linear law of changing magnetic field a gradient $K$ is $0.006+0.7 \mathrm{~T} / \mathrm{cm}$ and for exponential law of inhomogeneity a relative gradient $k$ is $0.05+1 \mathrm{~cm}^{-1}$. Polycrystalline aluminum samples were formed with help of respective templates and after bending were annealed, so the residual resistance ratio achieved $15 \cdot 10^{3}$. The measurements were made at $7=4.2 \mathrm{~K}$ in the regime of current supply using a reversal of current and magnetic field. The separation between the contacts for measuring signals along Hall direction (1-2-3-4-5, 1'-2'-3'-4'-5' etc.) was $1.5 \mathrm{~mm}$ and for the signals along the charge flow $\left(1-1^{\prime}-1^{m}-1^{m}-1^{m}, 2-2^{\prime}-2^{n}-2^{m}-2^{m+m}\right.$ etc.) $5 \mathrm{~mm}$. The allocation of electric potential along transport direction $\varphi(x, y=c o n s t)=\varphi(x)$ and along transversc direction $\Phi(x=$ const,$y)=\Phi(y)$ were measured on the sample surface.

\section{RESULTS AND ANALYSIS}

\section{Linearity of Transverse Magnetic Field Along Transport Direction}

The potential distributions have been mcasured for different magnetic fields and gradients. The data for uniform magnetic field have been obtained too. Notice that the dependencies $\phi(x)$ for various coordinates $y$ are not identical. These behaviors differ most significantly on opposite sides of sample (contacts $1-1$ '-... and $5-5$ '-...) (Fig.2). The recorded signals and potential differences between adjacent probes are
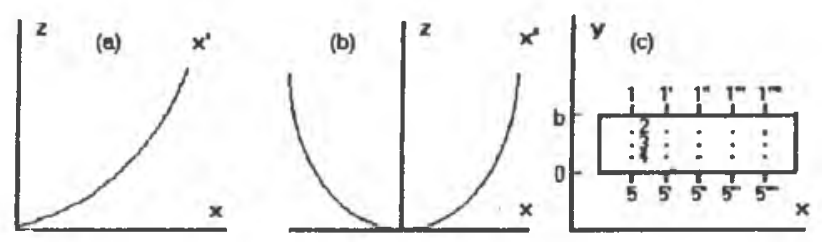

Figure 1. Experimental geometry: $(\mathrm{a}, \mathrm{b})$ - profiles of bent samples in plane $\mathrm{ZX}, z$ is a direction of external magnetic field $B, x^{\prime}$ is a direction of current $l_{\text {, (a) }}$ - asymmetric profile when a sign of magnetic field gradient is constant along transport, (b) - U-shaped profile when a gradient sign inverses in center of sample; (c) - scheme of potential probes disposition on sample face. 

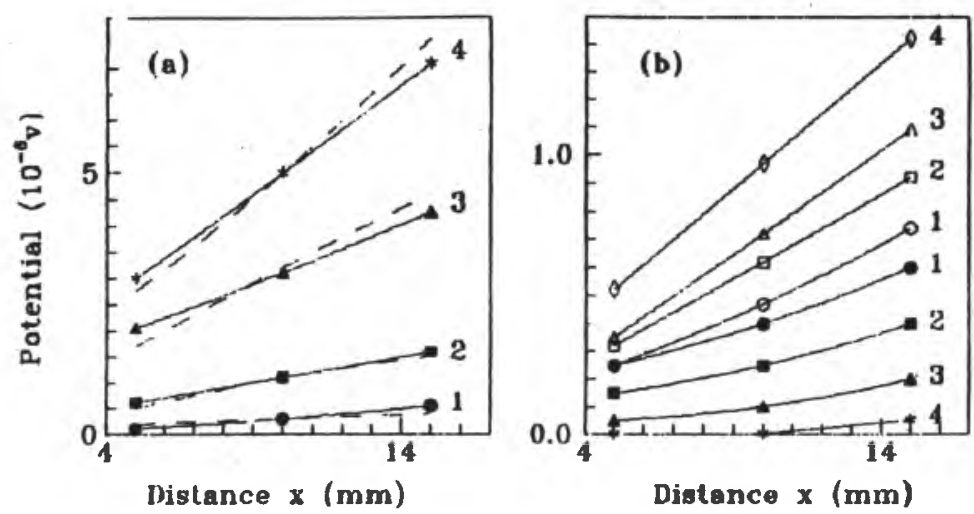

Figure 2. The potential $\varphi(x)$ on "strong" (a) and "weak" (b) sides for different $K$, T/cm: 0.04 (1), $0.14(2), 0.43(3), 0.7$ (4) and respective $B, T: 0.4,1,4,4.3,7.1$. Solid lines - experiment, dashed - theory; full symbols $-K^{\dagger} \neq 0$, emply $-K^{+}=0$.

large on one of sides (we'll call it as "strong" side) as shown in Fig. 2a. On the opposite side signals are small (wcill call this side as "weak") as shown in Fig. 2b. $W^{\prime}$ ith decreasing $K^{\top}$ the signals on "strong" side decrease and signals on "weak" side enlarge. Potential disttibution $\varphi(x)$ for the contacts situated in central region of a sample surface $\left(2-2,-\ldots, 3-3^{\prime}-\ldots, 4-4^{\prime}-\ldots\right)$ monotonically changes from one side to another. It should be mentioned that a non-linear dependence of potential on coordinate $y$ occurs (we shall return to this fact later). An inversion of current retains the allocation, that is a "strong" side remains a "strong" that and a "weak" side remains a "weak" that. A reversal of magnetic field stimulates a spatial inversion of potential distribution. The dependence $\Phi(x)$ for $1-1$ '-..., 2-2'-... transforms into

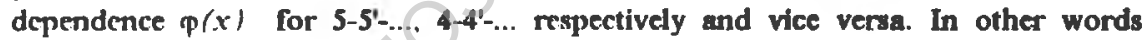
"strong" side dependence (ransforms to the "weak" that and on the contrary the "weak" transforms to the "strong". Analysis of this situation is based on the equation of electric fieid polential for isotropic dispersion law:

$$
\frac{\partial^{2} \varphi}{\partial x^{2}}+\frac{\partial^{2} \varphi}{\partial y^{2}}-\frac{2 \beta}{1+\beta^{2}} K \frac{\partial \varphi}{\partial x}+\frac{1-\beta^{2}}{1+\beta^{2}} K \frac{\partial \varphi}{\partial x}=0
$$

Here a dimensionless parameter $\beta=\omega, \tau$, $\omega$ is a cyclotron frequency, $\tau$ is a relaxation time, $K$ is a relative gradient which is represented in $\mathrm{cm}^{-1}$ and is connected with $K^{n}$ as follows:

$$
K=\frac{\partial \beta}{\partial x}=\frac{e \tau}{m c} K^{\prime}
$$

Boundary conditions are the continuity of charge on the surface and following the continuity of integral current through any cross-section of sample. Solution of Eq.(1) have been obtained by the method of separation of variables and may be represented:

$$
\Phi=I \beta \exp (K y)\{t \sigma[\exp (K b)-1]\}^{-1}
$$


Here $\sigma$ is a conductivity in zero magnetic field, $t$ is a sample thickness. Following Eg.(3) Hall current $j_{y}$ is absent in specimen volume and the curl character of current is realized by a redistribution of current density. That is current akinning occurs.

However the absence of Hall current in an analyzed approximation is not absolute in practice. Weak anisotropy of dispersion law of real metal (in our case it is aluminum) stimulates an appearance of non-zero electric field along magnetic field. Assuming the absence of $j_{z}$ both on the sample surface and in the volume as well as the uniformity of electric field along $z$ the Eq.(1) can be represented:

$$
\frac{\partial^{2} \Phi}{\partial x^{2}}+\frac{\partial^{2} \varphi}{\partial y^{2}}-\frac{\beta^{\prime}}{\beta} \frac{\partial \varphi}{\partial x}-\beta^{\prime}\left(1-\frac{1}{\beta}\right) \frac{\partial p}{\partial y}=0
$$

Using the method of iteration the next solution can be obtained:

$$
\Phi=C_{1}\left[\left(x \exp (K y)-K^{-1} \exp (-K x)-\left(\beta_{0}-K x+1\right)\left(\beta_{0} K\right)^{-1} \exp (-K x)\right]+C_{2}\right.
$$

Following this solution a small anisotropy of aluminum dispersion law admits the presence of Hall current under transverse inhomogeneous magnetic field changing by lincar law along transport direction.

\section{Exponential Type of Inhomogeneity of Mugnetic Field}

$\Lambda$ magnetoresistance of $\mathrm{Al}$ is known to be a linear function of magnetic field. In these conditions it is naturally to wait the cnhancement of Hall current influence on charge flowing. Exponential dependence of magnelic field inhomogeneity also may stimulate an additional redistribution of current. In view of these two factors (a linearity of magnetoresistance and an exponentiality of inhomogeneity) the results of potential and current redistribution are represented here. Experimental and analytical data are shown in fig. 3 where the dependencies of potential both for "strong" (Fig. 3a) and "weak" (Fig. 3b) sides are presented for different magnetic fields when $k=$ $0.3 \mathrm{~cm}^{-1}$. For comparison a potential dependence for a case of zero inhomogeneity when there is no any potential redistribution between two sides is exhibited in Fig. 3b
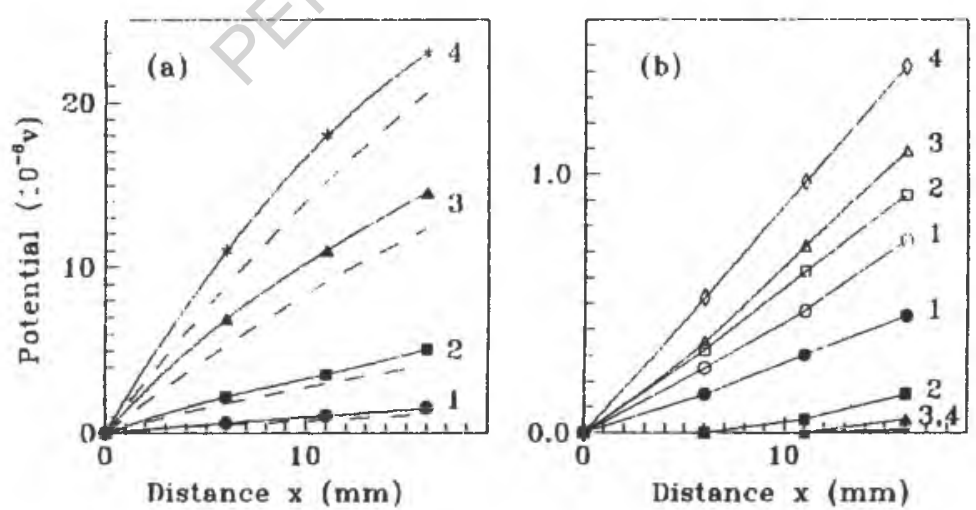

Figure 3. The potential $\varphi(x)$ on strong (a) and weak (b) sides for different $B, T: 0.35$ (1), 1.4 (2), 4.3 (3), 5.0 (4); solid lines - experiment, dashed - theory; full symbols $-k=0.3 \mathrm{~cm}^{-1}$, empty $-k=0$. 


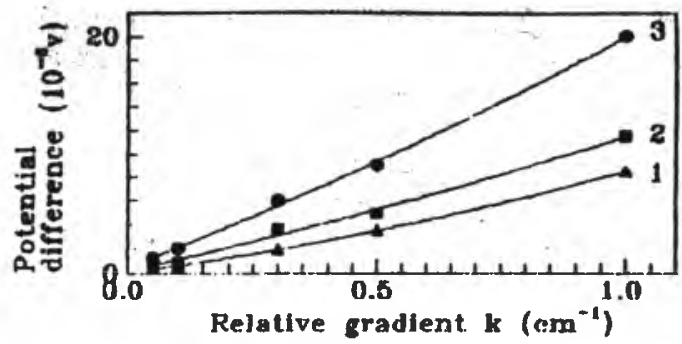

Figure 4. The potential difference $\Delta \varphi$ for different averaged fiedds $B, T: 1$ (1), 2.5 (2), 4 (3).

for the same external magnetic fields. As it is seen in Fig.3 and has been mentioned earlicr the effect of potential redistribution increase at magnetic field growth. The effect of redistribution increases with a rise of magnetic field gradient too. An influence of magnetic field inhomogencity on potential difference between two points $\varphi\left(x_{1}\right)-\varphi\left(x_{2}\right)$ is shown in Irig. 4 . Here a distance hetween probes in $5 \mathrm{~mm}$ and averaige external magnetic field in a center of segment is 4, 2.5 and I $T$. Thene dependencies respect in "strong" side of sample.

All basic relations between potentials for "strong" and "weak" sides are renlized for exponential type of inhomogencity. That is an inversion of current direction conscrves a potential distribution modulo but an inversion of sign of magnetic field gradient accompanies the reversal of magnetic ficld and it leads to exchange of redistribution between "strong" and "werk" sides. A transformation of potential distribution of "strong" side in "weak" that is confirmed by data displaced in Fig.5 for (1-shuped sample when $k=0.3 \mathrm{~cm}^{-1}$. In this geometry (Fig. 1b) an inveraion of sign of magnetic field gradient takes place in point $x=0$. Carriers move firstly dong increasing magnetic field and after point $x=0$ carriers move along decreasing magnetic ficld. So a transformation of a "strong"-like dependence in a "weak"-like dependence takcs place at $x=0$. Data measured on opposite sides of U-shaped

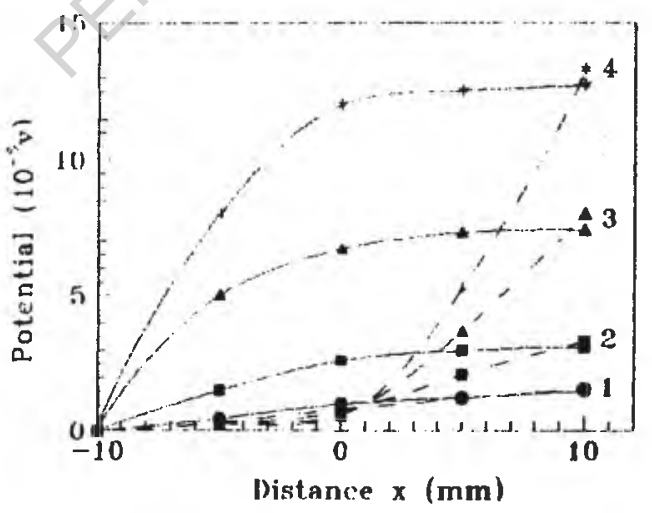

Figure 5. The potenlial $\varphi(x)$ for U-shaped sanple on opposite sides for diflenent $B, T: 0.14(1), 1.4(2)$, $4.3(3), 7.1(4) ; k=11.3 \mathrm{~cm}$ ': solid lines - one side, dashed - another. 
sample confirm that voltage signals are symmetrical for such geometry. $A$ distance between potential functions for opposite sides is a measure of inhomogeneity because for zero inhomogenclty these functions coincide in accordance with data represented in Fig.3b for homogeneous magnetic field.

It is dificult to evaluate potential and current redistribution under exponential type of inhomogeneity allowing for a linearity of magnetoresistance using the Eq.(1) because the conductivity tensor already corresponds to open Fermi surface. In this connection we apply the phenomenological expression for resistivity in magnetic field $\rho_{x a}=\rho_{s}(1+\lambda B)\left(\rho_{\text {is }}\right.$ is a saturation part of magnetoresistance and $\lambda$ is a coefficient of a non-saturation) and receive:

$$
\rho_{x x} \frac{\partial j_{x}}{\partial y}+p_{x y} \frac{\partial j_{y}}{\partial y}-\frac{\partial \rho_{x}}{\partial x} j_{x}-\rho_{y x} \frac{\partial j_{x}}{\partial x}-\frac{\partial \rho_{y y}}{\partial x} j_{y}-\rho_{y} \frac{\partial j_{y}}{\partial x}=0
$$

Further it is possible to neglect the dependence of component $\rho_{y y}$ on magnetic field bccause the experimental behavior of $\rho_{x x}$ as a function of $B$ in strong magnetic field curresponds to an open Fermi surface having narrow layer of open orbits ("corrugated cylinder" type). Allowing for $\lambda B$ is small as against unity and assuming $\partial j / \partial x=0$ ( the inhomogeneity is regular) one can get a current density distribution:

$$
j_{x}=I \rho_{y x}^{\prime} \exp \left(\rho_{y x}^{\prime} y^{\prime} \rho_{x x}\right)\left\{t p_{x x}\left[\exp \left(p_{y x}^{\prime} b / p_{x x}\right)-1\right]\right\}^{\prime} ; \rho_{y x}^{\prime}=\partial p_{y x} / \partial x
$$

The potentials $\varphi(x)$ are following:

$$
\varphi(x, y=b):: I \rho_{m} / t ; \Phi(x, y=0)=I \rho_{x x} \exp \left[\left(\cdots \rho_{y x}^{\prime} b / \rho_{x x}\right) \exp (k x)\right](k a b)^{-t}
$$

Thus the dependence of $\varphi(x)$ on "strong" side $(y=b)$ is governed by the spatial local valuc of Hall component of resistivity tensor. For present casc the parameters of matcrial are the ncxt: $\tau$ is of the order of $3 \cdot 10^{-10} \mathrm{sec}$. $\omega \tau$ achieve 350 in field $7 \mathrm{~T}, \rho_{n}=$ $4 \cdot 10^{10} \mathrm{62} \cdot \mathrm{cm}, \lambda=0.1 \mathrm{~T} \cdot 1$. Hall constant is equal $10^{-10} \mathrm{~m}^{3} / \mathrm{A} \cdot \mathrm{s}$. Using these data and following Eys.(2), (3), (5) and (7) the calculations of $\varphi(x)$ and $\varphi(y)$ have been made. Correlation between experimentul and analytical duta may be seen in Fig.2,3 and 6 .

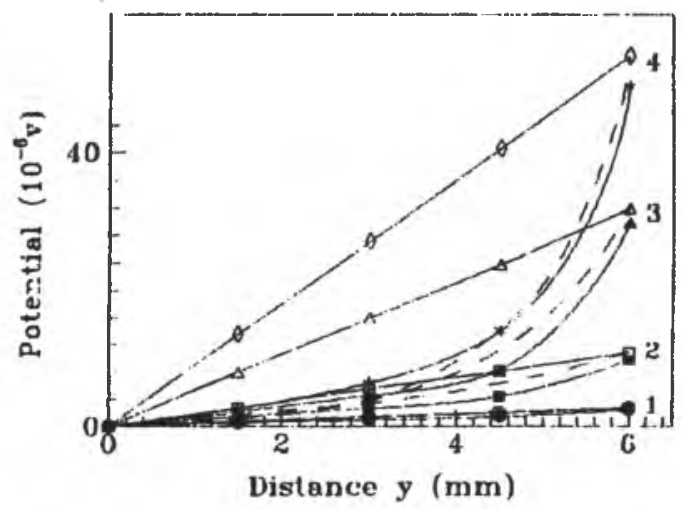

Figure 6. The potential $\Phi(y)$ for diferent $B, T: 0.35$ (1), $1.4(2), 4.3$ (3), 7.1 (4). Sulid lines - experiment. dashed - theory; full symbols $-k=0.3 \mathrm{~cm}^{\prime}$, empty $-k=0$. 
Total analyzis of situation gives some arguments to claim that a redistribution of potential measured on sample surface corresponds to its redistribution in whole volume. Respectively a current density through cross-section is inhomogencous. There is a high current density near "strong" side and a small current density near "weak" side of sample. Redistribution leads to decrease of cfloctive cross-section of sample and respective enhancement of magnetoresistance. The process of effective section diminishing is seen in Fig.6 where a potential distribution along transverse Hall coordinate $y$ is represented for both homogeneous and inhomogeneous cases. Uniform current" density $f_{x}$ corresponds to linear potential dependence on $y$. Inhomogencity leads to non-linear potential dependence and the degree of current skinning as well as cross section decrease may be estimated using the magnitudes of areas under $\varphi(y)$ for homogeneous and inhomogeneous curves.

\section{Some Experimental and Theoretical Relations. The Modes of Their Application}

The observed dependencies of potential on coordinates may be expressed in some relations. Thus for linear inhomogeneity the potential differences $\varphi_{I S} ; \varphi_{r^{\prime}} ; \varphi_{H}{ }$. : $\varphi_{\text {. }}$. (hete $p_{15}=\left(p_{1}-\left(p_{s}\right.\right.$ etc. $)$ can be represented:

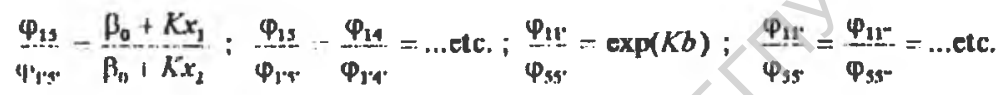

here $x_{1}$ and $x_{2}$ are the distances to respcutive pairs of contacts.

for intrinsic points $y_{1}$ and $y_{\text {: }}$

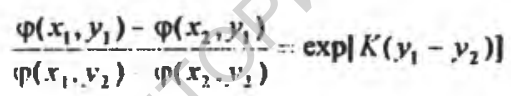

The analy sis of cxpontntial inhomogeneity detects the next ratio:

$$
\frac{\varphi_{11}}{\varphi_{1 \times}}=\exp \left|k\left(x_{1}-x_{2}\right)\right|
$$

The observed potential distribution and regarded relations give possibility to apply these phenomena in some passive devices of cryogenic electronics where a logarithmic and linear change of signals is required, for example to encrgize the input circuits of devices by the discretely variating voltsge. The use of signal ratios is also possibk. when several pairs of contacts are asked. Device on the basis of bent plate sample may be a logartthmic attenuator. Also this sample connected to the current gencrator may act as a converter transforming the current into voltage in accordance with linear and exponential law. Such situation was modeled during our experiment when the distribution of voltage differences was registered by potontiometer across the surface of investigated sample and the observed relations corresponded to analytically obtained data.

\section{CONCI,USION}

Using a new method of modeling the medium inhomogeneity the peculiarities of low temperature charge transport in pure aluminum under action of transverse 
inhomogeneous magnetic field have been inveatigated. The character of spatial dependence of potential of steady field was determined for both linear and exponential change of magnetic field along transport direction.

The andytical correlation between electric field, current skinning and the propertics of material has been eatablished on the base of approximations of electric field potentiality and charge continuity.

The correspondence of experimental and theoretical data each other for both types of inhomogeneity enables to advance some new principles of uses of discussed phenomena in cryogenic electronic devices and apparatus. Such devices may be organized in cryogenic systems having steady magnetic field.

This approach is suitable not only for pure aluminum conductor but for dilute aluminum alloys because this method is not confined by material purity. Moreover the used approximation of relaxation time is reasonable for high level of electronimpurity scattering so the temperature range is not restricted only by liquid helium.

\section{ACKNOWLLDGMENT}

This research is supported by liund of Fundamental Investigations of the Republic of Belarus (Grant No. F40-249). The authors are very grateful to Belarusian Soros Foundation for a chance of represcntation of this work at CEC/CMC'95 (Grant No. B95-04-1025-33).

\section{REFERLNCES}

1. R.T.Bate and A.C.Becr, Influence of conductivily gradicnts on galvanomagnetic effects in Semiconductors, J.of Appl.Phys. 32:800(1961),

2. J.B.Sampsell and J.C.Garland, Current distortion effects and linear magnetoresistance of inclusions in free-tectron metals, Phys. Rev. B. 13:583(1976),

3. V.I.Gostishchev, A.A.Drozd and S.F.Deın'yanov, Electric ficld distribution in aluminum in strong magnetic fields, Sov.J.Low Temp.Phys. 4:532(1978),

4. D.Stroud and F.P.Pan, Magnetoresistance and Hall coefficient in inhomogeneous metals, Phys.Rev.B. 20:455(1979),

5. Yu.A.Bogod, Vit.B.Krusovitsky and E.'I.Lemeshevskaya, Direct current skinning in inhomogeneous bismuth single crystals, Fiz.Nizk. Temp. 11:161(1985),

6. Yu.A.Bogod, Vit.B.Krasovitsky and F.T.L.emeshevskaya, Heat flux skinning in Bismuth, ibid. 13:1087(1987),

7. G.J.L.Bruls, J.Bass, A.P.vanGelder, H.van Kempen. and P.Wyder, Linear magnetoresistance due to sample thickness variations: Application to aluminum, Phys.Rev.B. 32:1927(1985)

8. H. Kaneko, and N.Yanagi, Enhancement of magnetoresistance due to Hall current in aluminum-copper composite, Cryogenics. 32:1114(1992),

9. O.R.Christianson and J.H.Parker, Anomalous magnetoresistance mechanisms, in: "Advances in Cryogenic Engineering (Materials)", Plenum Press, New York and I. ondon (1994), v.40A, p.425.

10. N.Yanagi, T.Mito, K.Takahata, M.Sakamoto, et al anomalous magnetoresistivity in 10-20 kA class aluminum-stabilized supcrconductors for the large helical device, ibid. p.459.

11. O.N.Mazurenko, V.R.Sobol, and A.A.Drozd, Magnetodynamics of conduction electrons in $\mathrm{A}$ in inhomogeneous field, Low Temp.Phys 21:59(1995). 